\title{
Quaternion-Kähler manifolds near maximal fixed point sets of $S^{1}$-symmetries
}

\author{
Aleksandra Borówka ${ }^{1}$ \\ Received: 29 May 2019 / Accepted: 28 September 2019 / Published online: 17 October 2019 \\ (c) The Author(s) 2019
}

\begin{abstract}
Using quaternionic Feix-Kaledin construction, we provide a local classification of quaternion-Kähler metrics with a rotating $S^{1}$-symmetry with the fixed point set submanifold $S$ of maximal possible dimension. For any real-analytic Kähler manifold $S$ equipped with a line bundle with a real-analytic unitary connection with curvature proportional to the Kähler form, we explicitly construct a holomorphic contact distribution on the twistor space obtained by the quaternionic Feix-Kaledin construction from these data. Conversely, we show that quaternion-Kähler metrics with a rotating $S^{1}$-symmetry induce on the fixed point set of maximal dimension a Kähler metric together with a unitary connection on a holomorphic line bundle with curvature proportional to the Kähler form and the two constructions are inverse to each other. Moreover, we study the case when $S$ is compact, showing that in this case the quaternion-Kähler geometry is determined by the Kähler metric on the fixed point set (of maximal possible dimension) and by the contact line bundle along the corresponding submanifold on the twistor space. Finally, we relate the results to the c-map construction showing that the family of quaternion-Kähler manifolds obtained from a fixed Kähler metric on $S$ by varying the line bundle and the hyperkähler manifold obtained by hyperkähler Feix-Kaledin construction from $S$ are related by hyperkähler/quaternion-Kähler correspondence.
\end{abstract}

Keywords Quaternion-Kähler manifold · Circle action · Maximal Legendrian submanifold · Quaternionic Feix-Kaledin construction · C-map

\section{Mathematics Subject Classification 53C26 -53C28}

\section{Introduction}

A classification of hyperkähler metrics with rotating $S^{1}$-symmetry near the fixed point submanifold $S$ of maximal dimension was provided by Feix [12] and, independently, by Kaledin [17]. They have shown that locally such metrics are uniquely determined by the real-analytic Kähler metric on $S$, and for any such metric the corresponding hyperkähler structure can be constructed on a neighbourhood of the zero section of the cotangent bundle of $S$. The twistorial construction of Feix has been generalized in [9], where a classification

Aleksandra Borówka

aleksandra.borowka@uj.edu.pl

1 Institute of Mathematics, Polish Academy of Sciences, ul. Śniadeckich 8, 00-656 Warszawa, Poland 
of quaternionic structures with rotating $S^{1}$-symmetry near the fixed point submanifold $S$ of dimension $n$ was given. In this case, $S$ is, so-called, real-analytic c-projective manifold with c-projective curvature of type $(1,1)$ and the structure is constructed on a neighbourhood of the zero section of the tangent bundle twisted by some unitary line bundle. Although considering a c-projective structure (instead of a Kähler metric) on $S$ is interesting mainly from non-metric point of view, introducing a twist by a line bundle with a connection allows obtaining nonzero scalar curvature analogues of hyperkähler metrics, namely quaternion-Kähler metrics. In [9] it is shown that all quaternionic structures admitting an $S^{1}$-action in the neighbourhood of the fixed point set $S$ of maximal dimension arise locally in this way, provided that the action on $S$ is not triholomorphic, i.e. the action is rotating complex structures from the quaternionic family.

The aim of this paper is to use the results from [9] to provide an analogous classification result for quaternion-Kähler metrics with rotating $S^{1}$-symmetry. We show that near the fixed point submanifold $S$ of dimension $n$ such metric is uniquely determined by the real-analytic Kähler metric on $S$ and a holomorphic line bundle on $S$ equipped with a real-analytic unitary connection with curvature proportional to the Kähler form. On the twistor space, the manifold $S$ corresponds to Legendrian submanifolds $S^{1,0} \cong S$ and $S^{0,1} \cong \bar{S}$ and the square of the line bundle is the contact line bundle restricted to $S^{1,0}$. When $S$ is compact, then by uniqueness of Hermite-Einstein metrics we deduce that the metric locally near $S$ is determined by the Kähler structure on $S$ and the contact line bundle. Note that in [9], the case when $S$ is KählerEinstein and the line bundle is a root of the canonical bundle is already studied; namely, it has been proved that then the constructed quaternionic manifold $M$ is quaternion-Kähler. The proof is non-explicit and involves studying hypercomplex cones (i.e. Swann bundles) over $M$ and Armstrong cones over $S$. In particular, the c-projective Armstrong cone over $S$ carries a natural Kähler metric if and only if $S$ is Kähler-Einstein which could suggest that this condition is necessary. In this paper, we show that this is not true: starting from any Kähler manifold with a holomorphic line bundle on $S$ with a real-analytic unitary connection such that its curvature is proportional to the Kähler form, we explicitly construct a holomorphic contact distribution on the twistor space. Therefore, the proposed construction gives abundance of local quaternion-Kähler metrics.

For a fixed Kähler structure on $S$ by varying the line bundle, we obtain a family of quaternion-Kähler manifolds which for the trivial line bundle degenerates to the unique hyperkähler metric. As already observed in [9] when $S$ is Kähler-Einstein, this is related to the hyperkähler/quaternion-Kähler correspondence which in mathematical language has been introduced by Haydys [14] motivated by a concept of the physicist's c-map. A further relation of this correspondence with the supergravity c-map [by allowing hyperkähler metrics of signature $(4,4 n)$ ] has been given by Alekseevsky et al. [1], and the twistorial description was given by Hitchin [15]. The manifolds appearing in the correspondence are equipped with a rotating $S^{1}$-symmetry, and it is assumed that the action is locally free. We show that our approach extends this correspondence to neighbourhoods of fixed point set of the $S^{1}$-action, provided that it is of maximal dimension. Note that for a fixed unitary connection on the line bundle over $S$, we obtain a one-parameter family of quaternion-Kähler metrics parametrized by the curvature. This agrees with the result obtained in [22] where they show that there is essentially one parameter of twists of hyperkähler metrics in hyperkähler/quaternion-Kähler correspondence.

The structure of the paper is as follows. In Sect. 2, we provide necessary background including the outline of the quaternionic Feix-Kaledin construction [9]. In Sect. 3, we give conditions on the data in the quaternionic Feix-Kaledin construction which are necessary to obtain as a result a quaternion-Kähler manifold with a rotating $S^{1}$-isometry and in Sect. 4, we 
prove that these conditions are sufficient. In Sect. 5, we discuss the case when $S$ is compact, proving in particular that the quaternion-Kähler structure on a neighbourhood of $S$ is fully encoded in the Kähler metric on $S$ and the contact line bundle on the twistor space restricted to the submanifold corresponding to $S$. Finally, in Sect. 6 we discuss examples and relation with the hyperkähler/quaternion-Kähler correspondence.

\section{Preliminaries}

\subsection{Complexification}

We start by giving a short summary about complexifications. For more detailed description, see $[8,9,19]$. For any real-analytic $m$-dimensional manifold $S$ using holomorphic extensions of real- analytic coordinates, we can construct its complexification, i.e. a complex manifold of complex dimension $m$ with a real structure whose fixed point set—-the real submanifold—is isomorphic to $S$. Similarly, we can use holomorphic extensions to extend real-analytic objects like bundles and connections from $S$ to its complexification. Note that locally near the real submanifold, any two complexifications are equal. If $S$ is itself a complex manifold, then there is a convenient model of complexification, namely the manifold $S^{\mathbb{C}}=S \times \bar{S}$, where $\bar{S}$ denote $S$ with the opposite complex structure, the real structure is the involution $(x, y) \mapsto(y, x)$ and its fixed point set is the diagonal. In this setting, if we consider complex local coordinates on $S$ and their complex conjugates $z_{1}, \bar{z}_{1}, \ldots, z_{n}, \bar{z}_{n}, m=2 n$, then the complexification is achieved by replacing coordinates $\bar{z}_{1}, \ldots, \bar{z}_{n}$ by independent coordinates $\tilde{z}_{1}, \ldots, \tilde{z}_{n}$, and then, the real submanifold is given by the equation $\tilde{z}_{i}=\bar{z}_{i}$. The manifold $S^{\mathbb{C}}$ carries two transverse foliations given by the product structure, and the leaves are isomorphic to $S$ and $\bar{S}$ correspondingly. We denote the foliations as the $(1,0)$-foliation and the $(0,1)$-foliation correspondingly and the leave spaces by $S^{0,1}$ and $S^{1,0}$.

For a holomorphic rank $k$ vector bundle $L$ on a complex manifold $S$, we will understand by its complexification $L^{\mathbb{C}}$ a holomorphic extension to a tubular neighbourhood of the diagonal in $S^{\mathbb{C}}$ of the bundle $L \otimes_{\mathbb{R}} \mathbb{C}$. The bundle $L^{\mathbb{C}}$ is a holomorphic bundle of rank $2 k$, and the holomorphic structure on $L$ induces its decomposition into a sum of two holomorphic bundles of rank $k$

$$
L^{\mathbb{C}}=L^{1,0} \oplus L^{0,1},
$$

such that $L^{1,0}$ is trivial along the leaves of the $(0,1)$-foliation and is the pullback of $L$ from $S^{1,0} \cong S$ and $L^{0,1}$ is trivial along the leaves of the $(1,0)$-foliation and is the pullback of $\bar{L}$ from $S^{0,1} \cong \bar{S}$. Any real-analytic connection $\nabla$ compatible with a holomorphic structure on $L$ gives connections on $L^{1,0}$ and $L^{0,1}$ trivial in the directions where the bundles are trivial, and one can show that the connections are flat in the other direction exactly when the curvature of $\nabla$ is of type $(1,1)$.

\subsection{Twistor theory for quaternionic manifolds}

Quaternionic manifold $M$ is a smooth manifold of dimension $4 n, n>1$ equipped with a rank 3 subbundle $Q \subseteq \operatorname{End}(T M)$ spanned locally by three anti-commuting almost complex structures satisfying quaternionic relations, together with a torsion-free connection $D$ with $D Q=0$, called a quaternionic connection. Inside $Q$, there is a 2 -sphere subbundle $\mathcal{Q}$ of almost complex structures and the total space of this bundle, denoted $Z$, is called the 
twistor space. It turns out that $Z$ is equipped with an almost complex structure $\mathcal{I}$ and the existence of torsion-free quaternionic connection is exactly the integrability condition for $\mathcal{I}$ (see [25]). Note that a torsion-free quaternionic connection is never unique (in fact, if one such connection exists, there is an affine space of such connections modelled on the space of 1 -forms on $M$ ), but one can show that the definition of $\mathcal{I}$ does not depend on the choice of $D$. Therefore, twistor spaces of quaternionic manifolds are complex manifolds of complex dimension $2 n+1$ and they are equipped with the following objects:

(i) family of projective lines with normal bundles $\mathbb{C}^{2 n} \otimes \mathcal{O}(1)$ called twistor lines,

(ii) a real structure $\tau$ without fixed points which is invariant on some twistor lines, called real twistor lines.

A twistor space fully determines quaternionic geometry on $M$, and it has been proved in [23] that any complex $(2 n+1)$-manifold $Z$ with properties (i) and (ii) is locally (near some fixed real twistor line) a twistor space of some quaternionic manifold, which as a smooth manifold is the moduli space of real twistor lines.

On a quaternionic manifold, one can always define a $Q$-Hermitian metric $g$; however, the Levi-Civita connection of such metric needs not be quaternionic (i.e. in general $D^{g}$ may not preserve the bundle $Q)$. If the Levi-Civita connection is quaternionic, then $(M, g, Q)$ is called quaternion-Kähler manifold. Quaternionic connections give $\tau$-invariant complex distributions on $Z$ (but not necessarily holomorphic, see [4], Sect. 5.3 for characterization of holomorphic distributions).

Definition 1 A holomorphic contact manifold $Z$ is a $(2 n+1)$-dimensional complex manifold equipped with a contact form $\theta \in \Gamma\left(T^{*} Z \otimes L\right)$ with values in some line bundle $L$ with the property

$$
\theta \wedge(\mathrm{d} \theta)^{n} \neq 0 .
$$

A distribution is called contact if it is the kernel of a contact form.

If a quaternionic connection is the Levi-Civita connection of a quaternion-Kähler metric, then the distribution is contact and holomorphic and in particular $Z$ is a holomorphic contact manifold ([24], Theorem 4.3).

Moreover, as shown in [20] Theorem 1.3, the following converse is also true.

Theorem 1 ([20]) Let $Z$ be a complex manifold of complex dimension $2 n+1$ satisfying the conditions (i), (ii) and

(iii) $Z$ is a holomorphic contact manifold with $\tau$-invariant contact distribution transversal to real twistor lines,

then $Z$ is locally a twistor spaces of a pseudo quaternion-Kähler manifold.

In this case, the line bundle $L$ is the vertical bundle of the twistor fibration (i.e. the bundle of tangent spaces to twistor lines; note that for general quaternionic manifold this bundle needs not to be holomorphic [18]) and it is the $(n+1)$ st root of the anti-canonical bundle of $Z$.

It is often convenient to keep in mind the complexified version of the twistor correspondence: the moduli space of all twistor lines is locally a $4 n$-dimensional complex manifold $M^{\mathbb{C}}$ with a real structure induced by $\tau$ whose fixed points correspond to $\tau$-invariant twistor lines: $M^{\mathbb{C}}$ is a complexification of the quaternionic manifold $M$. This makes sense as quaternionic manifolds are always real analytic. 
Definition 2 The incidence space for the complexified twistor correspondence is the space

$$
\mathscr{F}:=\left\{(z, u) \in Z \times M^{\mathbb{C}}: z \in u\right\} .
$$

The space $\mathscr{F}$ is a sphere bundle over $M^{\mathbb{C}}$, and it can be viewed as a complexification of the sphere bundle over $M$ of almost complex structures from the quaternionic structure. Moreover, a complexification of a quaternionic connection $D$ on $M$ induces a distribution on $\mathscr{F}$ which is the pullback from $Z$ to $\mathscr{F} \subset Z \times M^{\mathbb{C}}$ of the distribution given by $D$ on $Z$.

An important fact about complexifications of quaternionic manifolds is that locally the tangent space can be decomposed $T M^{\mathbb{C}}=\mathscr{E} \otimes \mathbb{C} \mathscr{H}$, where $\mathscr{E}$ and $\mathscr{H}$ are bundles associated with the standard complex representation of $S p(n)$ and $S p(1)$, respectively (see [25]). Many properties of quaternion-Kähler manifolds can be proved by studying these representations. In particular, the bundle $S^{2} \mathscr{E} \oplus S^{2} \mathscr{H}$ is naturally a subbundle of the bundle of 2-forms but also a subbundle of $\operatorname{End}(T M)$ and $S^{2} \mathscr{H}$ is spanned by the almost complex structures compatible with the quaternionic structure.

For $n=1$, we define quaternionic manifolds as self-dual conformal 4-manifolds and quaternion-Kähler as self-dual Einstein 4-manifolds and in this setting, the twistor correspondence extends to the dimension $n=1$.

\subsection{The quaternionic Feix-Kaledin construction}

The quaternionic Feix-Kaledin construction [9] (qFK in short) is a generalization of the hypercomplex and hyperkähler twistorial constructions of Feix [12]. Using real-analytic geometric data on a complex manifold $S$, it gives a quaternionic structure with a compatible $S^{1}$-action on a neighbourhood of the zero section of a twisted tangent bundle to $S$ and it has been proved that in this way, we can obtain any quaternionic structure with a quaternionic $S^{1}$-action locally near the fixed point set, provided that it is of maximal possible dimension and the action has no triholomorphic points. In this section, we will briefly summarize the construction from [9] as the main aim of this paper is to characterize when quaternionic manifolds obtained in this way are quaternion-Kähler. Note that we will present a simplified version of the construction by replacing a c-projective structure on $S$ by a fixed connection as only this case is relevant in the metric setting-the quaternion-Kähler metric restricts to a Kähler metric on S (see Proposition 1).

We will also outline some ideas from the original approach of Feix which will be relevant here.

Let $S$ be a complex manifold equipped with a torsion-free complex connection $D$ with curvature of type $(1,1)$ (for example, the Levi-Civita connection of a Kähler metric) and $\mathscr{L}$ be a holomorphic line bundle with a connection $\nabla_{\mathscr{L}}$ compatible with the holomorphic structure and with curvature of type $(1,1)$. As we have discussed in Sect. 2.1, the complexification of the bundle $\mathscr{L}$ decomposes into the sum of two line bundles $\mathscr{L}^{1,0}$ and $\mathscr{L}^{0,1}$ which are trivial along the leaves of the $(0,1)$ and the $(1,0)$-foliations, respectively, and equipped with the connections flat along the leaves of the $(1,0)$ and the $(0,1)$-foliation, respectively. Also, the connection $D^{c}$ on $T S^{\mathbb{C}}$ is flat along the leaves of the $(1,0)$ and the $(0,1)$-foliations, respectively.

Definition 3 Let $S$ be a smooth complex manifold of complex dimension $n$. The bundle $\mathcal{O}(1)$ over an open subset of $S$ is defined as a local $(n+1)$ st root of $\Lambda^{n} T^{1,0} S$, where $T^{1,0} S$ denote the holomorphic tangent bundle. Note that if $S=\mathbb{C P}^{n}$, then the bundle $\mathcal{O}(1)$ is defined globally and it is equal to the dual to the tautological bundle. 
Let us denote by $\mathscr{L}_{1,0}:=\mathscr{L}^{1,0} \otimes \mathcal{O}(1)$ and $\mathscr{L}_{0,1}:=\mathscr{L}^{0,1} \otimes \mathcal{O}(1)$ and by $\nabla$ the connections on $\mathscr{L}_{1,0}$ and $\mathscr{L}_{0,1}$ induced by $\nabla \mathscr{L}$ and $D$.

Over each leaf of the foliations, one defines $(n+1)$-dimensional complex vector space: in [12], this is the vector space of 'affine functions' along the leaf, i.e. these which along the leaf satisfy $D^{c} \mathrm{~d} f=0$, while in [9] it is the space of 'affine sections' of $\mathscr{L}_{1,0}$ and $\mathscr{L}_{0,1}$, respectively, defined as projections of parallel sections of a flat connection on the 1-jet bundle of $\mathscr{L}_{1,0}$ and $\mathscr{L}_{0,1}$ along the leaf. In our simplified case, the sections are tensor products of affine functions with parallel sections along the leaves for $\nabla$ on $\mathscr{L}_{1,0}$ and $\mathscr{L}_{0,1}$, respectively.

In this way, we define two rank $n+1$ holomorphic bundles over the leave spaces of the foliations (by requiring that a fibre over a fixed leaf is the space of affine sections along the leaf): $\operatorname{Aff}\left(\mathscr{L}_{1,0}\right)$ over $S^{0,1}$ and $\operatorname{Aff}\left(\mathscr{L}_{0,1}\right)$ over $S^{1,0}$ and we further define

$$
\mathscr{V}^{1,0}:=\left[\operatorname{Aff}\left(\mathscr{L}_{1,0}\right)\right]^{*} \otimes \mathscr{L}_{0,1}, \quad \mathscr{V}^{0,1}:=\left[\operatorname{Aff}\left(\mathscr{L}_{0,1}\right)\right]^{*} \otimes \mathscr{L}_{1,0} .
$$

For any $(x, \tilde{x}) \in S^{c}$ and $l \in\left[\left(\mathscr{L}_{1,0}\right)^{*} \otimes \mathscr{L}_{0,1}\right]_{(x, \tilde{x})}$ by evaluation, we can obtain an element of the fibre $\mathscr{V}_{\tilde{x}}^{1,0}$ defined by $s:=[f \mapsto f(x) \otimes l]$ (any $s \in \mathscr{V}_{\tilde{x}}^{1,0}$ is an element of the dual space to the space of affine sections of $\left(\mathscr{L}_{0,1}\right)^{*} \otimes \mathscr{L}_{1,0} \rightarrow S^{\mathbb{C}}$ along the leaf $\left.\tilde{x}\right)$ and analogously we can map elements of $\left[\left(\mathscr{L}_{0,1}\right)^{*} \otimes \mathscr{L}_{1,0}\right]_{(x, \tilde{x})}$ to elements of $\mathscr{V}_{x}^{0,1}$.

These evaluations induce maps $\phi_{1,0}$ from the total space of the bundle $\left(\mathscr{L}_{1,0}\right)^{*} \otimes \mathscr{L}_{0,1} \rightarrow$ $S^{\mathbb{C}}$ to $\mathscr{V}^{1,0}$ and $\phi_{0,1}$ from $\left(\mathscr{L}_{0,1}\right)^{*} \otimes \mathscr{L}_{1,0} \rightarrow S^{\mathbb{C}}$ to $\mathscr{V}^{0,1}$ which behave like a blow-down: they are biholomorphisms on the complements of the zero sections, and on the zero sections they contract leaves of one of the foliations; the images are cone subbundles of $\mathscr{V}^{1,0}$ and $\mathscr{V}^{0,1}$

Remark 1 In the Feix's case [12], the bundle $\left(\mathscr{L}_{1,0}\right)^{*} \otimes \mathscr{L}_{0,1}$ is trivial (the affine sections become affine functions) and one can find local coordinates $p_{1}, \ldots p_{n}, q_{1}, \ldots q_{n}$ on $S^{\mathbb{C}}$ such that the leaves of the $(1,0)$-foliation are given by setting $\left(q_{1}, \ldots q_{n}\right)$ to constant and $p_{1}, \ldots p_{n}$ are affine functions along the leaves. Moreover, in the hyperkähler case the complexified Kähler form on $S^{c}$ is equal to $\Sigma_{i=1}^{n} d p_{i} \wedge d q_{i}$. Then, in these coordinates the map $\phi_{1,0}$ is given explicitly by

$$
\left(p_{1}, \ldots p_{n}, q_{1}, \ldots q_{n}, \lambda\right) \mapsto\left(\lambda p_{1}, \ldots \lambda p_{n}, \lambda q_{1}, \ldots \lambda q_{n}, \lambda\right) .
$$

However, in general it is not possible to find such coordinates for $\phi_{1,0}$ and $\phi_{0,1}$ simultaneously.

The projective bundle $\mathbb{P}\left(\left[(\mathscr{L} \otimes \mathcal{O}(1))^{*}\right]^{\mathbb{C}}\right)=\mathbb{P}\left(\left[\mathscr{L}_{1,0}\right]^{*} \oplus\left[\mathscr{L}_{0,1}\right]^{*}\right)$ has canonical 0 and $\infty$ sections, and the corresponding affine bundles (obtained by removing one of the sections) are $\left(\mathscr{L}_{1,0}\right)^{*} \otimes \mathscr{L}_{0,1}$ and $\left(\mathscr{L}_{0,1}\right)^{*} \otimes \mathscr{L}_{1,0}$. This defines a gluing of $\mathscr{V}^{1,0}$ and $\mathscr{V}^{0,1}$ to a possibly non-Hausdorff complex manifold. However, we can choose open tubular neighbourhoods of the zero sections of $\mathscr{V}^{1,0}$ and $\mathscr{V}^{0,1}$ containing the images of $\phi_{1,0}$ and $\phi_{0,1}$ such that the gluing is a Hausdorff manifold $Z$.

It turns out that $Z$ satisfies the properties of quaternionic twistor space: the real structure is induced by the real structure $\tau$ given by the complexification and the images of fibres of $\mathbb{P}\left(\left[(\mathscr{L} \otimes \mathcal{O}(1))^{*}\right]^{\mathbb{C}}\right)$ are twistor lines. The twistor lines obtained in this way are the twistor lines corresponding to $S^{\mathbb{C}} \subset M^{\mathbb{C}}$, where $M$ is the quaternionic manifold obtained from $Z$. To simplify the notation, we make the following definition.

Definition 4 The twistor lines which are images of fibres of $\mathbb{P}\left(\left[(\mathscr{L} \otimes \mathcal{O}(1))^{*}\right]^{\mathbb{C}}\right)$ are called canonical twistor lines and the fibres over the real submanifold $S \subset S^{\mathbb{C}}$ are called canonical real twistor lines. The canonical twistor lines correspond to points in a neighbourhood of $S$ in the submanifold $S^{\mathbb{C}} \subset M^{\mathbb{C}}$. 
The construction can be summarized by the following diagram.

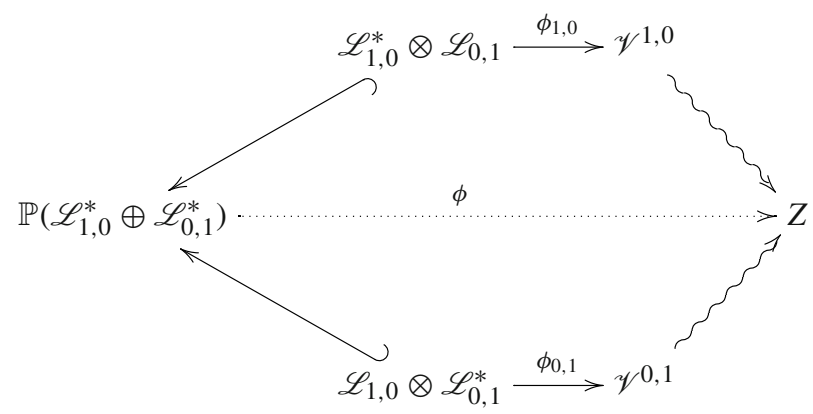

The scalar multiplication (and its inverse) in the fibres induces a holomorphic $\tau$-invariant $\mathbb{C}^{*}$-action on $Z$ fixing the zero sections of $\mathscr{V}^{1,0}$ and $\mathscr{V}^{0,1}$, tangent to canonical twistor lines and transversal to twistor lines near the canonical real twistor lines. Therefore, it corresponds to a quaternionic $S^{1}$-action on $M$ such that $S \subset M$ is the fixed point set.

Definition 5 A fixed point of a quaternionic $S^{1}$-action on a quaternionic manifold is called triholomorphic if the induced action on the twistor space fixes all points on the corresponding twistor line.

The points on the fixed point set $S \subset M$ of the action obtained by qFK are not triholomorphic. In [9], it is shown that the twistor space of any quaternionic manifold with such an $S^{1}$-action can be obtained locally in this way: on the twistor space $Z$, the fixed point set corresponds to a submanifold of complex dimension $n$ with two components on which the corresponding $\mathbb{C}^{*}$-action has constant single weight equal to 1 and one can deduce that $Z$ is of the form as in the quaternionic Feix-Kaledin construction.

Note that if $M$ is a quaternion-Kähler manifold, then any isometry is also a symmetry of quaternionic structure-see [3] Sect. 5 (but the converse is not true). Hence, we will use terms $S^{1}$-symmetry and $S^{1}$-isometry in this setting interchangeably.

\subsection{Quaternion-Kähler moment map}

For any $S^{1}$-symmetry (i.e. isometry) on a quaternion-Kähler manifold $M$, one can define its moment map ([13]) in the following way. Let $\omega_{i}(\cdot, \cdot):=g\left(I_{i} \cdot, \cdot\right)$, where $I_{1}, I_{2}, I_{3}$ is a local quaternionic base. Then, the 4-form $\Omega:=\left(\omega_{1}\right)^{2}+\left(\omega_{2}\right)^{2}+\left(\omega_{3}\right)^{2}$ is defined globally, is non-degenerate and does not depend on the choice of the local quaternionic bases. The moment map of the action is a section of $S^{2} \mathscr{H}$ (where $T M^{\mathbb{C}}=\mathscr{E} \otimes_{\mathbb{C}} \mathscr{H}$ and $S^{2} \mathscr{H}$ is a subbundle of 2-forms, see Sect. 2.2) satisfying

$$
\mathrm{d} \mu=i_{X} \Omega,
$$

where $X$ is the Killing field of the action. The moment map is unique, and on the twistor space it corresponds to the section $\theta(\hat{X})$ of the contact line bundle $L$ (see [15] Sect. 4.3), where $\hat{X}$ is the vector field on $Z$ corresponding to $X$ and $\theta$ is the contact form. In [7] Proposition 3.3, it is shown that away from $\mu^{-1}(0)$, the moment map induces a distinguished (up to a sign) integrable complex structure $\pm I$ invariant under the $S^{1}$-action. From the twistorial point of view, the complex structure corresponds to one of the two components of the zero set of the twistorial moment map $\theta(\hat{X})$ : along real twistor lines, the bundle $L$ (in which $\theta$ takes the values) is the bundle $\mathcal{O}(2)$; hence, $\theta(\hat{X})$ along any real twistor line either vanishes or has 
exactly two zeros. The first possibility is excluded on the complement of $\mu^{-1}(0)$; hence, locally we have two components of the zero set of $\theta(\hat{X})$.

If $J$ is any almost complex structure from the quaternionic structure anti-commuting with $I$ (possibly defined only locally), then we have that $\mathcal{L}_{X} J=K$, where $K=I J$.

\section{The necessary condition for the existence of a quaternion-Kähler metric}

In the literature, one can find two different definitions of totally complex submanifolds of quaternion-Kähler manifolds. One of them is connected purely with the underlying quaternionic structure (see [9]), while the other gives also a condition for the metric (see [6]). Recall that $\mathcal{Q}$ is the 2-sphere subbundle of $Q$ (which is the bundle of almost complex structures forming the quaternionic structure).

Definition 6 A submanifold $S$ of $(M, Q)$ is totally complex if there exists a section $I$ of $\left.\mathcal{Q}\right|_{S}$ (in particular $I^{2}=-i d$ ) such that the following two conditions are satisfied:

(i) $I(T S) \subseteq T S$ (so that $I$ is an almost complex structure on $S$ );

(ii) for all $J$ anti-commuting with $I, J(T S) \cap T S=0$.

A submanifold $S$ of $(M, Q)$ is metrically totally complex if it satisfies the conditions (i), (ii) and the following condition (iii):

(iii) $J(T S)$ is $g$-orthogonal to $T S$ for any $J$ anti-commuting with $I$.

Note that as it is shown in [5] Theorem 5.2, the above conditions imply that $J$ is integrable; hence, $S$ is a complex submanifold of $M$.

Let $Z$ be a twistor space of a quaternion-Kähler manifold $M$ with an $S^{1}$-action which is an isometry, has no triholomorphic points and its fixed point set component $S$ is of the maximal possible dimension (i.e. of complex dimension $n$ ). Then, locally near $S$, the manifold $M$ can be obtained by the quaternionic Feix-Kaledin construction (see Sect. 2.3) and the moment map has no zeros as the action is rotating the twistor lines corresponding to $S$. We restrict $(Z, M, S)$ such that they globally arise by the construction. As described in Sect. 2.3, in this case, the twistor space is a gluing of tubular neighbourhoods of zero sections of two holomorphic rank $(n+1)$ vector bundles $\mathscr{V}^{1,0}$ and $\mathscr{V}^{0,1}$. The zero sections of the bundles correspond to $(S, I)$ and $(S,-I)$, where $I$ is the complex structure on $S$ fixed by the action. The bundle structure on $\mathscr{V}^{1,0}$ and $\mathscr{V}^{0,1}$ is given by the holomorphic $\mathbb{C}^{\times}$-action on $Z$ corresponding to the $S^{1}$-action. As $(M, g)$ is quaternion-Kähler, the distribution induced by the Levi-Civita connection on $Z$ is holomorphic and contact and we denote by $D^{g}$ the quaternionic-Kähler connection on $M$ and by $\theta$ the contact form. We abuse the notation and denote the holomorphic contact distribution on $Z$ (i.e. the kernel of $\theta$ ) also by $D^{g}$.

As explained in Sect. 2.4, any quaternion-Kähler manifold with a compatible circle action on the complement of the zero set of the moment map admits a distinguished (up to a sign) $S^{1}$-invariant compatible integrable complex structure $I$, which is defined by the zero set of the twistorial moment map. In our case, this complex structure is an extension of the complex structure on $S$.

Proposition 1 Suppose that the manifold $M$ obtained by the quaternionic Feix-Kaledin construction from manifold $S$ is quaternion-Kähler such that the obtained $S^{1}$-action is an isometry. Then, $S$ is s Kähler and metrically totally complex. 
Proof As $S$ is contained in the fixed point set of the action, it follows from [7] Proposition 3.5 that $S$ is Kähler. Hence, by [6] Theorem 1.12 it metrically totally complex.

The complex structure $I$ on $Z$ is a holomorphic smooth divisor $\mathscr{D}^{1,0}$ which contains the zero section of the bundle $\mathscr{V}^{1,0}$.

Lemma 1 Let $M$ be a quaternion-Kähler manifold obtained by qFK construction such that the natural $S^{1}$-action is an isometry. Then, the divisor $\mathscr{D}^{1,0}$ on $Z$ corresponding to the distinguished (up to a sign) $S^{1}$-invariant complex structure I defined by the moment map is a rank $n$ subbundle of the vector bundle $\mathscr{V}^{1,0}$.

Proof Recall that the $S^{1}$-action on $M$ gives a $\mathbb{C}^{\times}$-action on $Z$ which on $\mathscr{V}^{1,0}$ is the scalar multiplication in the fibres. The divisor $\mathscr{D}^{1,0}$ represents the unique (up to a sign) complex structure $I$ which is $S^{1}$-invariant; therefore, $\mathscr{D}^{1,0}$ is $\mathbb{C}^{\times}$-invariant. Hence, as $\mathscr{D}^{1,0}$ is smooth, it is a holomorphic rank $n$ subbundle of $\mathscr{V}^{1,0}$ transversal to canonical real twistor lines (see Definition 4).

As a result, we have that $(M, I) \cong \mathscr{D}^{1,0}$ and this isomorphism is $S^{1}$-equivariant.

Since $Z$ is a holomorphic contact $(2 n+1)$ manifold, through any point of $Z$ locally there are Legendrian $n$-dimensional holomorphic submanifolds tangent to the contact distribution. Let $u_{x, \bar{x}}$ be the real twistor line through the zero of $\mathscr{D}_{\bar{x}}^{1,0}$ for $\bar{x} \in S^{0,1}$. The twistor line $u_{x, \bar{x}}$ passes through the zero section of $\mathscr{V}^{0,1}$ which we denote by $\underline{\infty}$ and $u_{x, \bar{x}} \backslash \underline{\infty} \subset \mathscr{V}_{\bar{x}}^{1,0}$ (as this is canonical real twistor line-see Sect. 2.3).

Lemma 2 For any $\bar{x} \in S^{0,1}$ and any $z \in u_{x, \bar{x}} \backslash \underline{\infty}$, there exists a unique Legendrian submanifold contained in $\mathscr{V}_{\bar{x}}^{1,0}$. Moreover, the Legendrian submanifolds with this property are affine hyperplanes.

Proof Inside the fibre $\mathscr{V}_{\bar{x}}^{1,0}$, there is a cone $\mathcal{C}_{\bar{x}}$ given by the union of the canonical twistor lines which are images of fibres of the Lagrangian submanifold $S_{\bar{x}}^{1,0} \subset S^{\mathbb{C}}$ (see Definition 4). In other words, $S_{\bar{x}}^{1,0}$ is the leaf of the $(1,0)$-foliation through $\bar{x}$. As the curvature of the connection $D^{g}$ is of type $(1,1)$ on $S \subset M$, the complexified connection is flat along $S_{x}^{0,1}$. Hence, it is flat on the preimage of $\mathcal{C}_{\bar{x}}$ in the incident space $\mathscr{F}$ (see Definition 2) and the Legendrians $\mathscr{W}_{z}$ for $\left.z \in u_{x, \bar{x}} \backslash \underline{\infty}, \underline{0}\right\}$ are the images of the parallel sections. (The parallel section corresponding to $z \in \underline{0}$ is contracted to a point.)

Now consider the limit of the tangent spaces $W_{z}$ to $\mathscr{W}_{z}$ along $u_{x, \bar{x}}$ as $z \rightarrow 0 \in \mathscr{V}_{\bar{x}}^{1,0}$. We have that $W_{z}$ are Legendrian subspaces equal to $\operatorname{ker}(\theta)_{x} \cap \mathscr{V}_{\bar{x}}^{1,0}$; hence, the limit is a well-defined Legendrian subspace $W$ equal to $\operatorname{ker}(\theta)_{0} \cap \mathscr{V}_{\bar{x}}^{1,0}$ - the dimension of $W$ is equal to $n$ as along $u_{x, \bar{x}}$ the space $\mathscr{V}_{\bar{x}}^{1,0}$ is transversal to the contact distribution.

As the contact distribution on $Z$ is $\mathbb{C}^{\times}$-invariant and the action preserves the fibres, we deduce that the family of submanifolds $\mathscr{W}_{z}$ for $z \in u_{x, \bar{x}} \backslash\{\underline{\infty}, \underline{0}\}$ is $\mathbb{C}^{\times}$invariant. Hence, the spaces $W_{z}$ tangent to the Legendrians in $\mathscr{V}_{\bar{x}}^{1,0}$ are constant along the orbit of the $\mathbb{C}^{\times}$-action (note that $\mathscr{V}_{\bar{x}}^{1,0}$ is a vector space so the tangent bundle is trivial) and equal to the limit as $z \rightarrow 0 \in \mathscr{V}_{\bar{x}}^{1,0}$ which is $W$. Hence, the Legendrians $\mathscr{W}_{z}$ for $z \in u_{x, \bar{x}} \backslash\{\underline{\infty}, \underline{0}\}$ are affine hyperplanes modelled on the vector space $W$. As the vector subspace $W$ of $\mathscr{V}_{\bar{x}}^{1,0}$ is the limit of Legendrian submanifolds $\mathscr{W}_{z}$ as $z \rightarrow 0 \in \mathscr{V}_{\bar{x}}^{1,0}$, it is also Legendrian submanifold. 
Remark 2 Note that in the above Lemma, we have shown that for any $\bar{x} \in S^{0,1}$ the space $Z \cap \mathscr{V}_{\bar{x}}^{1,0}$ is foliated by the Legendrians. Moreover, rewriting $\operatorname{ker}(\theta)=D^{g}$ we have that $\left.D^{g} \cap \mathscr{V}_{\bar{x}}^{1,0}\right|_{0}=W$.

We can obtain an analogous result for the space $Z \cap \mathscr{V}_{x}^{0,1}$, where $x \in S^{1,0}$.

In Lemma 1, we have shown that $D^{g}$ on $Z$ is tangent to $\left.I\right|_{S}$ and clearly it cannot be tangent to the whole $\mathscr{D}^{1,0}$ (as it is contact). However, the following is true.

Proposition 2 For any $\bar{x} \in S^{0,1}$, the fibre $\left(\mathscr{D}_{\bar{x}}^{1,0}, I\right)$ is tangent to the contact distribution $D^{g}$; hence, it is metrically totally complex submanifold, and in particular Kähler.

Proof We aim to show that $\mathscr{D}_{\bar{x}}^{1,0}=W$. Both submanifolds are vector spaces of the same dimension. On the twistor space $Z$, the submanifold $\mathscr{D}^{1,0}$ is defined as one of the two components of the zero set of the twistorial moment map. The moment map is equal to $\theta(\hat{X})$, where $\hat{X}$ is a generator of the $\mathbb{C}^{\times}$-action on $Z$. Hence, on $\mathscr{D}^{1,0}$ we have that $\hat{X} \in \operatorname{ker} \theta$ belongs to $D^{g}$ and this condition characterizes $\mathscr{D}^{1,0}$ in some neighbourhood of 0 . Let $l \subset W$ be a vector line. Then, for any point $w \in l \backslash\{0\}$, the vector $\hat{X}_{w}$ is tangent to $l$ (as this is the generator of the scalar multiplication). Hence, $\hat{X}_{w} \in \operatorname{ker}\left(\theta_{w}\right)$ as it is tangent to a Legendrian submanifold. Therefore, $l \backslash\{0\}$ (and hence, $l$ ) is contained in $\mathscr{D}_{\bar{x}}^{1,0}$ which completes the proof.

Theorem 2 Let $M$ be a quaternion-Kähler $4 n$-manifold with an $S^{1}$-isometry which has a component of the fixed point set $S$ of dimension $2 n$ and such that the action on $S$ has no triholomorphic points. Then, locally near $S$, the twistor space $Z$ of $M$ can be obtained by the quaternionic Feix-Kaledin construction, the submanifold used in the construction is Kähler, and the connection on the line bundle is unitary and has curvature proportional to the Kähler form.

Proof As discussed in Sect. 2.3, the twistor space $Z$ in this case is obtained by gluing open subsets of two vector bundles $\mathscr{V}^{1,0}$ and $\mathscr{V}^{0,1}$. The bundle $\mathscr{V}^{1,0}$ is the bundle over the leaf space of the $(1,0)$-foliation such that the fibre over a leaf is the space dual to the vector space of 'affine sections' of $\mathscr{L}^{1,0} \otimes\left(\mathscr{L}^{0,1}\right)^{*}$ (see Sect. 2.3). By Lemma 1 , for any $\bar{x} \in S^{0,1}$ the manifold $\mathscr{D}_{\bar{x}}^{1,0}$ is a vector subspace of $\mathscr{V}_{\bar{x}}^{1,0}$; hence, we can find an affine section $f_{\bar{x}}$ along the leaf $\bar{x}$ of the $(1,0)$-foliation such that is given by the equation $s\left(f_{\bar{x}}\right)=0$, for $s \in \mathscr{V}_{\bar{x}}^{1,0}$.

Moreover, by Lemma 2 and Proposition 2, the Legendrians contained in $\mathscr{V}_{\bar{x}}^{1,0}$ are affine subspaces modelled on $\mathscr{D}_{\bar{x}}^{1,0}$; hence, the leaves of the foliation by the Legendrians are given by the equations $s\left(f_{\bar{x}}\right)=a$ for $a \in \mathbb{C}$. As the map $\phi_{1,0}$ is the evaluation map, the inverse images of the Legendrians in $\left(\mathscr{L}^{1,0}\right)^{*} \otimes \mathscr{L}^{0,1}$ are $\left[a f_{\bar{x}}\right]^{-1}$. An analogous argument gives a foliation by Legendrians of the fibres of the bundle $\mathscr{V}^{0,1}$ defining corresponding sections $\tilde{f}_{x}$ over the leaves of the $(0,1)$-foliation. Hence, for points from the intersection of $\mathscr{V}^{0,1} \cap \mathscr{V}^{1,0} \subset Z$, (which coincide with the images of the maps $\phi_{1,0}$ and $\phi_{0,1}$ on the complement of the zero sections) through any point we constructed two Legendrians which are transversal, and hence, the tangent spaces to the Legendrians span the contact distribution.

The pullback by $\phi_{1,0}$ of the contact distribution is the distribution on $\left(\mathscr{L}^{1,0}\right)^{*} \otimes \mathscr{L}^{0,1}$ spanned by the tangent spaces to $\left[a f_{\bar{x}}\right]^{-1}$ and $b \tilde{f}_{x}$. The contact distribution on $Z$ preserves the real structure which in qFK is induced on $Z$ by the complexification. Therefore, sections $\left[a f_{\bar{x}}\right]^{-1}$ and $b \tilde{f}_{x}$ intersecting in a fibre of $\left(\mathscr{L}^{1,0}\right)^{*} \otimes \mathscr{L}^{0,1}$ over the real submanifold $S \subset$ $S^{\mathbb{C}}$ are conjugated. Hence, the obtained distribution is defined by a complexification of a connection on the bundle $\left(\mathscr{L}^{1,0}\right)^{*} \otimes \mathscr{L}^{0,1}$ over $S$ with curvature of type $(1,1)$. We have 
shown that the parallel sections along the leaves are inverses of non-vanishing affine sections along the leaves, and hence, the connection on $\left(\mathscr{L}^{1,0}\right)^{*} \otimes \mathscr{L}^{0,1}$ that they generate comes from the connection $\nabla$ on $\mathscr{L} \otimes \mathcal{O}(1)$ used in the construction.

By [6] (see Eq. 2.2 and remark after Theorem 1.12), along any metrically totally complex submanifold with the complex structure $I$ of a quaternion-Kähler manifold $(M, g)$, the circle bundle of almost complex structures orthogonal to $I$ is $D^{g}$ invariant and there exists a 1-form $\gamma$ on $M$ such that along this submanifold we have

$$
D^{g} J=\gamma K, \quad D^{g} K=-\gamma J,
$$

where $J, K$ are orthogonal. Moreover, in this setting the curvature of the connection on the circle bundle is equal to $d \gamma$ and is proportional to $g(I \cdot, \cdot)$.

Recall that the manifold $M$ admits a unique complex structure invariant under the $S^{1}$ action; hence, globally on $M$ we have a well-defined circle bundle of almost complex structures orthogonal to $I$. This bundle is a smooth submanifold of $Z$ and, by Eq. 2, after restriction to $S \subset M$ it is preserved by the contact distribution (but globally this does not hold). Complexifying, we obtain a circle bundle over $S^{\mathbb{C}} \subset M^{\mathbb{C}}$ on the incidence space (see Definition 2), which is preserved by the complexified connection. Taking the projection to $Z$ from the incidence space, we obtain a set $\mathscr{U}$ and it is easy to see using the flatness of the connection along the leaves of the $(1,0)$ and $(0,1)$-foliation that this is the union of Legendrians contained in the fibres of $\mathscr{V}^{1,0}$ through the points on the canonical twistor lines belonging to the circle bundle. Note that we can analogously describe the set $\mathscr{U}$ using Legendrians in fibres of bundle $\mathscr{V}^{0,1}$ and that this implies that the both approaches give the same set.

The inverse image of $\mathscr{U}$ is a circle bundle over $S^{\mathbb{C}}$ preserved by the connection which defines a complexified unitary structure on $\left(\mathscr{L}^{1,0}\right)^{*} \otimes \mathscr{L}^{0,1}$. This proves that the connection $\nabla$ on $\mathscr{L} \otimes \mathcal{O}(1)$ is unitary. (Note that the induced connection on $\left(\mathscr{L}^{1,0}\right)^{*} \otimes \mathscr{L}^{0,1}$ restricted to the real submanifold $S$ is always unitary; hence, to conclude that $\nabla$ itself is unitary, we needed a complexified unitary structure.)

The condition on the curvature follows from the fact that the curvature of the circle bundle over any metrically totally complex submanifold is proportional to $g(I \cdot, \cdot)$; hence, along $S$ it is proportional to the Kähler form.

Remark 3 We have shown that the complex structure induced by the quaternion-Kähler moment map, in quaternionic Feix-Kaledin construction, corresponds to subbundles of the bundles $\mathscr{V}^{1,0}$ and $\mathscr{V}^{0,1}$ defined as annihilators of the parallel sections for $\nabla^{c}$ along the leaves of the $(1,0)$ and $(0,1)$-foliations on $S^{c}$, respectively. It is straight forward to see that through quaternionic Feix-Kaledin construction, we always obtain in this way a complex structure, provided that the c-projective structure is generated by a real-analytic connection with curvature of type $(1,1)$ : in this case, any such a connection in the c-projective class equips $M$ with a complex quaternionic structure. Such manifolds have been studied recently by N. Hitchin [16].

\section{Construction of a holomorphic contact distribution on the twistor space}

The aim of this section is to prove that the necessary conditions for the twistor space obtained by qFK to be a twistor space of quaternion-Kähler manifold are sufficient: assuming that the initial data in the construction satisfy the necessary conditions given in Theorem 2, we will explicitly construct a holomorphic distribution on the twistor space which is invariant under 
the real structure and the $\mathbb{C}^{\times}$-action and transversal to canonical real twistor lines. Moreover, provided that the initial Kähler metrics is positive definite we will show that the underlying quaternion-Kähler metric is positive definite.

\subsection{Standardization}

As explained in Remark 1, the method in [12] involves a local identification of each of the bundles of affine functions (i.e. halves of the twistor space) with a standard flat model. Note that this identification can be done for both bundles, but the identifications are not compatible with each other unless the structure is flat. We will show a similar result for qFK in the case when the c-projective structure is generated by a real-analytic connection with type $(1,1)$ curvature.

Let $(S, D)$ be a complex manifold of real dimension $2 n$ with a real-analytic connection with curvature of type $(1,1)$ and $\nabla_{\mathscr{L}}$ be a real-analytic connection compatible with the holomorphic structure with type $(1,1)$ curvature on a holomorphic line bundle $\mathscr{L}$. By $\nabla$, we denote the tensor product connection on $\mathscr{L} \otimes \mathcal{O}(1)$, where the connection on $\mathcal{O}(1)$ is induced by $D$. Consider the quaternionic Feix-Kaledin construction with $\left(S,[D]_{c}, \mathscr{L}, \nabla \mathscr{L}\right)$, where $[D]_{c}$ is the c-projective structure generated by $D$.

Lemma 3 The triples $\left(\left(\mathscr{L}_{1,0}\right)^{*} \otimes \mathscr{L}_{0,1}, \phi_{1,0}, \mathscr{V}^{1,0}\right)$ and $\left(\left(\mathscr{L}_{0,1}\right)^{*} \otimes \mathscr{L}_{1,0}, \phi_{0,1}, \mathscr{V}^{0,1}\right)$ can be locally standardized such that they are holomorphically equivalent to the standardized halves of the hypercomplex Feix construction.

Proof We will prove this for $\left(\left(\mathscr{L}_{1,0}\right)^{*} \otimes \mathscr{L}_{0,1}, \phi_{1,0}, \mathscr{V}^{1,0}\right)$, as the proof for the other half is analogous. As explained by Feix (see [12], Lemma 2), we can pick local holomorphic coordinates $p_{1}, \ldots, p_{n}, q_{1}, \ldots q_{n}$ of $S^{c}$ such that the leaves of the $(1,0)$-foliation are defined setting $q_{1}, \ldots q_{n}$ to be constants (in particular, we can take $\left(q_{1}, \ldots q_{n}\right)=$ $\left.\left(\tilde{z}_{1}, \ldots, \tilde{z}_{n}\right)\right)$ and $p_{1}, \ldots, p_{n}$ are affine functions along the leaves. Choose a section $f_{0}$ of $\left(\left(\mathscr{L}_{1,0}\right)^{*} \otimes \mathscr{L}_{0,1}\right)$ which is parallel along the leaves of the $(1,0)$-foliation. Then, the affine sections of $\mathscr{L}_{1,0} \otimes\left(\mathscr{L}_{0,1}\right)^{*}$ along the leaves are generated by $f_{0}^{-1}, f_{0}^{-1} p_{1}, \ldots, f_{0}^{-1} p_{n}$; and hence, $\left(q_{1}, \ldots q_{n}, f_{0}^{-1}, f_{0}^{-1} p_{1}, \ldots, f_{0}^{-1} p_{n}\right)$ are local coordinates on $\mathscr{V}^{1,0}$. Let us trivialize $\left(\left(\mathscr{L}_{1,0}\right)^{*} \otimes \mathscr{L}_{0,1}\right)$ using $f_{0}$, so that it becomes the trivial bundle $S^{c} \times \mathbb{C}$ with the fibre coordinate $t$. Then, it is immediate to check that the map $\phi_{1,0}$ in these coordinates is given by the formula

$$
\left(p_{1}, \ldots, p_{n}, q_{1}, \ldots q_{n}, t\right) \mapsto\left(t, t p_{1}, \ldots, t p_{n}, q_{1}, \ldots q_{n}\right)
$$

which is the form required by the Feix's standardization.

\subsection{Construction of the contact distribution}

First, we discuss the formula of the connection in local coordinates. Let $S$ be a real-analytic Kähler manifold and $\mathscr{L} \otimes \mathcal{O}(1)$ be a holomorphic line bundle on $S$ with a real-analytic holomorphic unitary connection $\nabla$ whose curvature is a scalar multiple $c$ of the Kähler form $\omega$. Then, in a local trivialization the connection takes the form

$$
\nabla=d+c \sum_{i=1}^{n} a_{i}(z, \bar{z}) \mathrm{d} z_{i},
$$


where $d \sum_{i=1}^{n} a_{i} \mathrm{~d} z_{i}=\sum_{i=1}^{n} \mathrm{~d} a_{i} \wedge \mathrm{d} z_{i}=\omega$. The condition that $\nabla$ is unitary means that there exists a positive-valued function $h(z, \bar{z})$ such that

$$
c \sum_{i=1}^{n} a_{i} \mathrm{~d} z_{i}=\partial \log h=\sum_{i=1}^{n} \frac{\partial \log h}{\partial z_{i}} \mathrm{~d} z_{i} .
$$

In this setting, $\eta:=\log h$ is a Kähler potential for $\omega$ as $\mathrm{d} \partial=\bar{\partial} \partial$.

Now consider the complexified picture. The corresponding connection

$$
d+c \sum_{i=1}^{n} a_{i}(z, \tilde{z}) \mathrm{d} z_{i}
$$

on $\mathscr{L}_{1,0}$ is flat along the leaves of the $(1,0)$-foliation and the function $e^{-\eta(z, \tilde{z})}=[h(z, \tilde{z})]^{-1}$ is parallel along the leaves of the $(1,0)$-foliation. Observe also that the corresponding connection on $\mathscr{L}_{0,1}$ is $d+c \Sigma_{i=1}^{n} \tilde{a}_{i}(z, \tilde{z}) \mathrm{d} \tilde{z}_{i}$, where $\tilde{a}_{i}(z, \tilde{z}):=a_{i}(\tilde{z}, z)$ is a complexification of $a_{i}(\bar{z}, z)$; hence, the connection on $\mathscr{L}_{1,0} \otimes\left[\mathscr{L}_{0,1}\right]^{*}$ is given in this trivialization by the formula:

$$
d+c \sum_{i=1}^{n} a_{i} \mathrm{~d} z_{i}-c \sum_{i=1}^{n} \tilde{a}_{i} \mathrm{~d} \tilde{z}_{i}
$$

Lemma 4 Consider $q F K$ with $S$ Kähler and $(\mathscr{L} \otimes \mathcal{O}(1), \nabla)$ such that $\nabla$ is unitary and its curvature is proportional to the Kähler form. The holomorphic distribution given by a complexification of the connection $\nabla$ on $\mathbb{P}\left(\left([\mathscr{L} \otimes \mathcal{O}(1)]^{*}\right)^{\mathbb{C}}\right)=\mathbb{P}\left(\mathscr{L}_{1,0}^{*} \oplus \mathscr{L}_{0,1}^{*}\right)$ extends via the map $\phi$ to a holomorphic contact distribution on $Z$.

Proof We will show that the distribution extends to $Z \cap \mathscr{V}^{1,0}$. The proof for the other half is analogous. The connection distribution on $\left[\mathscr{L}_{1,0}\right]^{*} \otimes \mathscr{L}_{0,1}$ is given as the kernel of the following 1-form

$$
\varphi:=\mathrm{d} f-f c \sum_{i=1}^{n} a_{i} \mathrm{~d} z_{i}+c f \sum_{i=1}^{n} \tilde{a}_{i} \mathrm{~d} \tilde{z}_{i},
$$

where $a_{i}, \tilde{a}_{i}$ are defined above and $f$ is a fibre coordinate. Now observe that as $(1,0)$-foliation is by definition given by $\tilde{z}=$ const and $\mathrm{d} \tilde{a}_{i} \wedge \mathrm{d} \tilde{z}_{i}=-\omega$ we have that $\tilde{a}_{i}$ are affine along the leaves (see [12]), and hence, the standardizing coordinates $p_{i}$ from Lemma 3 can be taken to be equal $\tilde{a}_{i}$. Moreover, we can set the standardizing trivialization $f_{0}$ to be equal $e^{\eta}$. By setting $t:=e^{-\eta} f$, we pass to the standardizing trivialization of $\mathscr{L}_{1,0} \otimes\left[\mathscr{L}_{0,1}\right]^{*}$ and the connection $\nabla$ in these coordinates is equal

$$
d\left[e^{\eta} t\right]-c e^{\eta} t \sum_{i=1}^{n} a_{i} \mathrm{~d} z_{i}+c e^{\eta} t \sum_{i=1}^{n} \tilde{a}_{i} \mathrm{~d} \tilde{z}_{i}=e^{\eta}\left[\mathrm{d} t+c t \sum_{i=1}^{n} \frac{\partial \eta}{\partial \tilde{z}_{i}} \mathrm{~d} \tilde{z}_{i}+c t \sum_{i=1}^{n} \tilde{a}_{i} \mathrm{~d} \tilde{z}_{i}\right] .
$$

As $h$ is a positive function on $S$, its logarithm $\eta$ is real valued on $S$, and hence, $[\partial \eta](z, \bar{z})=$ $[\bar{\partial} \eta](\bar{z}, z)$, and therefore, $\frac{\partial \eta}{\partial \tilde{z}_{i}}=\tilde{a}_{i}$. Using the standardized coordinates on $\mathscr{V}^{1,0}$, we get that the form $e^{-\eta} \varphi$ extends to $\mathscr{V}^{1,0}$ and the obtained distribution is contact which completes the proof.

Remark 4 The contact form on a twistor space has values in a line bundle which after restriction to any of the real twistor lines is isomorphic to $\mathcal{O}(2)$. In our setting, this is represented by 
the fact that although the distribution given by $\varphi$ extends to $Z$, the form $\varphi$ does not. Indeed, using the notation from the proof of Lemma 4 , the form $e^{-\eta} \varphi$ extends to one half of the twistor space (namely to $\mathscr{V}^{1,0}$ ) while $e^{\eta} \varphi$ extends to the other one $\left(\mathscr{V}^{0,1}\right)$.

Theorem 3 Let $S$ be a real-analytic Kähler manifold, $\mathscr{L}$ a holomorphic line bundle on $S$ with a real-analytic connection $\nabla_{\mathscr{L}}$ such that the connection $\nabla$ induced on $\mathscr{L} \otimes \mathcal{O}(1)$ is a unitary connection with curvature equal to $c \omega$, where $\omega$ is the Kähler form and $c$ is constant. Then, the twistor space $Z$ obtained by $q F K$ from $\left(S, \omega, \mathscr{L}, \nabla_{\mathscr{L}}\right)$ is a holomorphic contact manifold which is a twistor space of a quaternion-Kähler manifold $M$ with scalar curvature equal to $2 c$. Moreover, the $S^{1}$-action on $M$ coming from the construction is an isometry.

Proof By Lemma 4, we have already proved that $Z$ is a holomorphic contact manifold. By construction, the obtained distribution is preserved by the real structure on $Z$ and transversal to canonical real twistor lines. The existence of such contact distribution on the twistor space is equivalent to the existence of a pseudo quaternion-Kähler metric. As $S$ is metrically totally complex, we have that for $J$ anti-commuting with $I$ the tangent bundle splits $T M=$ $T S \oplus J T S$ and this splitting is $g$-orthogonal. Moreover, we have also that $g(J \cdot, J \cdot)=g(\cdot, \cdot)$; hence, the positive definiteness of the metric follows directly from the positive definiteness of the initial Kähler metric. In the proof of Theorem 2, we have shown that $2 c \omega$ is the curvature of the circle bundle of orthogonal complex structures over the metrically totally complex manifold $S \subset M$. By [6], this implies that $2 c$ is the scalar curvature of the underlying quaternion-Kähler manifold.

Finally, it is straightforward to check that the $\mathbb{C}^{\times}$-action on $Z$ given by scalar multiplication in fibres preserves the contact distribution; hence, it corresponds to an $S^{1}$ isometry on $M$.

Remark 5 Similarly, as in the hyperkähler case of Feix, we can generalize this argument pseudo quaternion-Kähler metric — by the construction we obtain pseudo quaternion-Kähler metrics with signature $(2 p, 2 q)$ when starting from a Kähler metrics with signature $(p, q)$.

\section{Gluing along the submanifold}

First, note that the qFK construction ([9]) is global in some neighbourhood of $S$ : although during the construction we have to restrict several times to local neighbourhoods, they are always tubular neighbourhoods of the whole manifold $S$. (And on the level of twistor space, it is a tubular neighbourhood of the union of canonical real twistor lines-i.e. the one that correspond to $S$.) Hence, in particular if $S$ is compact Kähler manifold and $\mathscr{L}$ are a holomorphic line bundle on $S$ with a real-analytic holomorphic connection $\nabla$ with curvature of type $(1,1)$, then we can obtain a quaternionic manifold $M$ which contains the whole $S$ as the zero set of an $S^{1}$-symmetry and any two quaternionic manifolds which on the fixed point set $S$ induce the same structure (i.e. the Kähler metric and the line bundle with a connection) are locally equivalent near $S$. Also observe that the construction of the contact distribution in Sect. 4.2 glues uniquely along $S$ (viewed as the zero section in $\mathscr{V}^{1,0}$ and $\mathscr{V}^{0,1}$, respectively), and hence, we obtain the following proposition.

Proposition 3 Suppose that $S$ is a compact Kähler manifold with a holomorphic line bundle $(\mathscr{L} \otimes \mathcal{O}(1), \nabla)$ with a connection satisfying the assumptions of Theorem 3 . Then, there exist (not necessarily compact) quaternion-Kähler manifold $M$ containing $S$ as a component of the fixed point set of an isometric $S^{1}$-action with no triholomorphic points on $S$. Moreover, all such $M$ are equivariantly isomorphic near $S$. 
However, we can prove a stronger fact. Note that as the contact line bundle (i.e. the bundle in which the contact form takes the values) is the vertical bundle of the twistor fibration, along the zero section of $\mathscr{V}^{1,0}$ it is isomorphic to $\left.\mathscr{L}_{1,0} \otimes\left[\mathscr{L}_{0,1}\right]^{*}\right|_{S}$, where $S$ is the diagonal in $S^{\mathbb{C}}$. By definition of the complexification we have that along the diagonal $S \subset S^{\mathbb{C}}$, we have

$$
\left.\overline{\mathscr{L}_{1,0}}\right|_{S}=\left.\mathscr{L}_{0,1}\right|_{S}
$$

and, as the bundle $\mathscr{L} \otimes \mathcal{O}(1)$ is unitary (by Theorem 2), we also have that

$$
\left.\mathscr{L}_{1,0}\right|_{S}=\left.\overline{\mathscr{L}}_{1,0}{ }^{*}\right|_{S}
$$

We summarize this observation with the following corollary.

Corollary 1 if $S$ is a fixed point set component of a quaternion-Kähler manifold with an isometric $S^{1}$-action with no triholomorphic points on $S$, then the bundle $\mathscr{L} \otimes \mathcal{O}(1)$ used in the construction must be a square root of a holomorphic bundle with a connection which is globally defined on $S$.

Proof Recall that $\mathscr{L} \otimes \mathcal{O}(1)=\mathscr{L}_{1,0} \oplus \mathscr{L}_{0,1}$. As $\left.\overline{\mathscr{L}_{1,0}}\right|_{S}=\left.\mathscr{L}_{0,1}\right|_{S}$ and $\left.\overline{\mathscr{L}_{1,0}}\right|_{S}=\left.\mathscr{L}_{0,1}\right|_{S}$, the contact line bundle along the zero section of $\mathscr{V}^{1,0}$ (and $\mathscr{V}^{0,1}$ ) is equal to $\mathscr{L}_{1,0}^{\otimes 2}$ which finishes the proof.

Now we will show that the converse is also true.

Theorem 4 Suppose that $(S, \omega)$ is a real-analytic Kähler manifold equipped with a holomorphic line bundle $\mathfrak{L}$ with a real-analytic unitary connection on $S$ which has curvature $C \omega$ proportional to the Kähler form. Let $\mathscr{L}$ be such that $\mathscr{L} \otimes \mathcal{O}(1)$ is a local square root of $\mathfrak{L}$ and $\nabla$ is the induced connection on $\mathscr{L}$. Then, there exists a quaternion-Kähler manifold $M$ with scalar curvature $C$ equipped with an isometric $S^{1}$-action such that $S$ is a component of the fixed point set and $M$ locally arises by the quaternionic Feix-Kaledin construction from $\mathscr{L}$ with the induced connection. Moreover, such a manifold is unique near $S$.

Proof By Theorem 4, such $M$ exists locally near any open subset of $S$ where the bundle $\mathscr{L}$ is defined.

First, observe that if $\mathscr{L}$ is a unitary line bundle, then the complexified Hermitian product on $\mathscr{L}^{\mathbb{C}}=\mathscr{L}^{1,0} \oplus \mathscr{L}^{0,1}$ over $S^{c}$ gives a paring between $\mathscr{L}^{1,0}$ and $\mathscr{L}^{0,1}$, and hence, the bundle $\mathscr{L}^{1,0} \otimes \mathscr{L}^{0,1}$ is trivial and as a consequence the bundles $\left(\mathscr{L}_{1,0}\right)^{\otimes k} \otimes\left(\mathscr{L}_{0,1}\right)^{\otimes k}$ are canonically trivial for any $k$ rational. Hence, the bundle $\left(\mathscr{L}_{1,0}\right)^{*} \otimes \mathscr{L}_{0,1}$ is canonically isomorphic with $\left(\mathscr{L}_{0,1}\right)^{2}$.

Therefore, we obtain a bundle $\mathscr{F}$ of projective lines over an open neighbourhood of the diagonal $S$ in $S^{\mathbb{C}}$ by gluing globally the vector bundles $\mathscr{L}_{0,1}^{2}$ and $\mathscr{L}_{1,0}^{2}$ using the canonical triviality of $\mathscr{L}_{1,0} \otimes \mathscr{L}_{0,1}$ in the following way:

$$
\begin{aligned}
\mathscr{L}_{0,1}^{2} & \cong\left[\left(\mathscr{L}_{1,0}\right)^{*} \otimes \mathscr{L}_{0,1}\right] \otimes\left[\mathscr{L}_{1,0} \otimes \mathscr{L}_{0,1}\right] \\
& \cong\left[\left(\mathscr{L}_{0,1}\right)^{*} \otimes \mathscr{L}_{1,0}\right]^{*} \otimes\left[\mathscr{L}_{1,0} \otimes \mathscr{L}_{0,1}\right]^{*} \cong\left(\mathscr{L}_{1,0}^{2}\right)^{*}
\end{aligned}
$$

In this setting, locally $\mathscr{F}$ is equal to $\mathbb{P}\left(\mathscr{L}_{1,0}^{*} \oplus \mathscr{L}_{0,1}^{*}\right)$. Denote by $\mathscr{V}_{e}^{1,0}$ the dual to the bundle of affine sections of $\left(\mathscr{L}_{0,1}^{2}\right)^{*}$ along the leaves of the $(1,0)$-foliation and by $\mathscr{V}_{e}^{0,1}$ the dual to the bundle of affine sections of $\left(\mathscr{L}_{1,0}^{2}\right)^{*}$ along the leaves of the $(0,1)$-foliation. Then, we obtain the following diagram: 


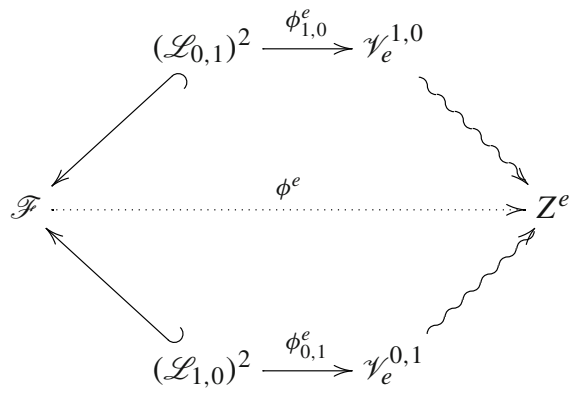

The bundle $\mathscr{V}_{e}^{1,0}$ is defined globally over $S^{0,1}$, and using again canonical triviality of $\mathscr{L}_{1,0} \otimes \mathscr{L}_{0,1}$ we get that it is canonically isomorphic to $\mathscr{V}^{1,0}$ over any subset of $S^{1,0}$ where $\mathscr{V}^{1,0}$ is defined. Similarly, $\mathscr{V}_{e}^{0,1}$ is an extension of $\mathscr{V}^{0,1}$ to the bundle globally defined over $S^{0,1}$. Therefore, Diagram 3 is an extension of Diagram 1 from Sect. 2.3 summarizing qFK. Hence, the obtained manifold $Z^{e}$ is locally a twistor space arising by qFK from $(\mathscr{L}, \nabla)$ and by construction and by Theorem 4.2, it is immediate to see that it is a twistor space of a quaternion-Kähler manifold with the required properties which contains the whole manifold $S$.

The uniqueness of $M$ near $S$ follows from Theorem 2 and from [9] (Theorem 4): suppose that $M$ is a quaternionic manifold with the required properties containing the whole manifold $S$. Then, the incidence space of the twistor fibration restricted to the canonical twistor lines is locally near the canonical real twistor lines a bundle of projective lines over a complexification of $S$ with two distinguished disjoint sections corresponding to the opposite complex structures. Moreover, the contact distribution defines on the affine parts of the bundle a complexification of the connection used locally in the construction. In particular, after the restriction of the bundle to the diagonal we obtain the connection used in the construction on $\left(\mathscr{L}_{1,0}\right)^{*} \otimes \mathscr{L}_{0,1} \cong\left(\mathscr{L}_{0,1}\right)^{2}$ which is globally defined on $S$. Using local equivalence of complexifications, it follows that the affine parts of the incidence space must be locally isomorphic to the affine parts of $\mathscr{F}$ which are glued together in the same way. It follows that the quaternion-Kähler manifolds are locally equivalent near $S$.

Remark 6 In the proof of the above theorem instead of using the triviality of $\mathscr{L}_{1,0} \otimes \mathscr{L}_{0,1}$, we can use the triviality of $\left(\mathscr{L}_{1,0} \otimes L^{1,0}\right) \otimes\left(\mathscr{L}_{0,1} \otimes L^{0,1}\right)$ for any $L$ unitary defined globally over $S$-then $\mathscr{F}$ will be constructed as gluing of different bundles, but the resulting space as well as the bundles $\mathscr{V}_{e}^{1,0}$ and $\mathscr{V}_{e}^{0,1}$ will remain unchanged. In particular, in the case when $S=\mathbb{C P}^{n}$, we can use this observation to identify the bundles $\mathscr{V}^{1,0}$ and $\mathscr{V}^{0,1}$ : the bundle $\mathcal{O}(1)$ (see Definition 3 ) is well defined over the whole $\mathbb{C P}^{n}$, and the bundle of affine sections of $\mathcal{O}(1,0)$ along the leaves of the $(1,0)$-foliation is trivial. Now the bundle of affine sections of $\mathscr{L}_{1,0} \otimes \mathscr{L}_{0,1}^{*}$ is isomorphic to the bundle of affine sections of $\mathcal{O}(1,1) \otimes\left(\mathscr{L}_{0,1}^{*}\right)^{2}$, and taking the dual we get that $\mathscr{V}^{1,0}$ is isomorphic to $\mathcal{O}(-1) \otimes\left(\mathscr{L}_{0,1}\right)^{2} \otimes \mathbb{C}^{2 n+1}$. We will use this approach in discussion of examples in the next section.

We can further summarize the obtained results in the following theorem.

Theorem 5 Let $S$ be a real-analytic compact Kähler manifold of complex dimension n and L a line bundle on $S$ such that it admits a real-analytic unitary connection such that the curvature is proportional to the Kähler form. Then, there exist possibly non-compact holomorphic contact $(2 n+1)$-manifold $Z$ which is a twistor space of a quaternion-Kähler manifold and contains $S$ as Legendrian submanifold and such that $L$ is the contact line bundle restricted 
to $S$. Moreover, $Z$ admits a holomorphic $\mathbb{C}^{\times}$-action fixing only $S$ and $\bar{S}$ corresponding to an isometric $S^{1}$-action.

Any two holomorphic contact manifolds with the above properties are biholomorphic on some neighbourhood of the set of all real twistor lines through $S$.

Proof The condition on curvature implies that the unitary connection on $L$ is HermiteEinstein. By Kobayashi-Hitchin correspondence, Hermite-Einstein connections on any holomorphic line bundle over compact Kähler manifold are unique, and hence, the contact line bundle determines the unique unitary connection with curvature proportional to the Kähler form which by Theorems 3 and 4 completes the proof.

\section{Examples and applications}

In this section, we will discuss the examples of $\mathbb{C P}^{2 n+1}$ and the flag manifold $F_{1, n+1}\left(\mathbb{C}^{n+2}\right)$ which already appeared in [9] and see how this fits into the setting of Remark 6.

Example 1 Let $S=\mathbb{C P}^{n}$ with the standard Kähler structure. As discussed in [9], by taking $\mathscr{L}$ trivial (which means that $\mathscr{L}_{1,0}=\mathcal{O}(1)$ ) by qFK we obtain $Z=\mathbb{C P}^{2 n+1}$ which is the twistor space of the quaternionic projective space.

In this case, we consider affine sections of $\mathcal{O}(1,-1)$ along the leaves of the $(1,0)$-foliation, and hence, by Remark 6 it should mean that in the construction we obtain $Z$ by gluing two copies of the bundle $\mathcal{O}(1) \otimes \mathbb{C}^{n+1}$ over $\mathbb{C P}^{n}$ using the blow-down from $\mathbb{P}(\mathcal{O}(-1,0) \oplus$ $\mathcal{O}(0,-1))$. Indeed, fixing a projective $n$-space $S$ in $\mathbb{C P}^{2 n+1}$ there is a natural embedding of the bundle $\mathcal{O}(1) \otimes \mathbb{C}^{n+1}$ into $\mathbb{C P}^{2 n+1}$ such that $S$ is the zero section: denoting points in $\mathbb{C P}^{2 n+1}$ by $\left[x_{0}: \ldots: x_{n}: y_{0}: \ldots: y_{n}\right]$, we can take $S$ to be given by the equation $\left(y_{0}, \ldots, y_{n}\right)=0$ and $\bar{S}$ by $\left(x_{0}, \ldots, x_{n}\right)=0$, and then, on the complement of $\bar{S}$, the space $Z$ can be identified as the total space of $\mathcal{O}(1) \otimes \mathbb{C}^{n+1}$. The blow-down map from the affine part $\mathcal{O}(-1,1)$ over $S^{\mathbb{C}}=\mathbb{C P}^{n} \times \mathbb{C P}^{n}$ is given explicitly by

$$
l \otimes\left[y_{0}, \ldots, y_{n}\right] \in \mathcal{O}(1,-1)_{\left[x_{0}: \ldots: x_{n}\right] \times\left[y_{0}: \ldots: y_{n}\right]} \mapsto\left[x_{0}: \ldots: x_{n}: y_{0}: \ldots: y_{n}\right],
$$

where $l\left(x_{0}, \ldots, x_{n}\right)=1$ and we get the analogous result for the other half of the twistor space. Note that the $\mathbb{C}^{\times}$-action on $\mathbb{C} \mathbb{P}^{2 n+1}$ given by

$$
\lambda\left[x_{0}: \ldots: x_{n}: y_{0}: \ldots: y_{n}\right] \mapsto\left[\lambda x_{0}: \ldots: \lambda x_{n}: \lambda^{-1} y_{0}: \ldots: \lambda^{-1} y_{n}\right]
$$

fixes exactly submanifolds $S$ and $\bar{S}$ and acts as the scalar multiplication in these fibres.

Example 2 Let $S=\mathbb{C P}^{n}$ with the standard Kähler structure and let $\mathscr{L}$ be $\mathcal{O}\left(-\frac{1}{2}\right)$ (hence, $\left.\mathscr{L}_{1,0}=\mathcal{O}\left(\frac{1}{2}\right)\right)$. As discussed in [9] in this case by qFK, we obtain the flag manifold $Z=$ $F_{1, n}\left(\mathbb{C}^{n+2}\right)$ which is the twistor space of the Grassmannian $G r_{2}\left(\mathbb{C}^{n+2}\right)$. By Remark $6, Z$ is the gluing of two trivial rank $n+1$ bundles using the blow-downs from $\mathcal{O}(-1,0)$ and $\mathcal{O}(0,-1)$, respectively, and the canonical triviality of $\mathcal{O}\left(\frac{1}{2}, \frac{1}{2}\right)$.

Indeed, let $\mathbb{C}^{n+2}$ with standard coordinates $\left(p, q_{0}, \ldots, q_{n}\right)$ be equipped with the standard Hermitian product and the $\mathbb{C}^{\times}$-action $\left(\lambda^{-1} p, \lambda q_{0}, \ldots \lambda q_{n}\right)$ and denote by $a$ the line defined by the equation

$$
a:=\left\{\left(p, q_{0}, \ldots, q_{n}\right): q_{0}=\cdots=q_{n}=0\right\} .
$$

Then, the real structure on $Z$ is defined by $\perp$ and the $\mathbb{C}^{\times}$-action on $\mathbb{C}^{n+2}$ induces a $\mathbb{C}^{\times}$-action on $Z$ fixing two $\perp$-conjugated submanifolds of $Z$ isomorphic to $\mathbb{C P}^{n}$; namely,

$$
S^{1,0}:=\left\{(l, K) \in F_{1, n}\left(\mathbb{C}^{n+2}\right): l=a\right\} \quad \text { and } \quad S^{0,1}:=\left\{(l, K) \in F_{1, n}\left(\mathbb{C}^{n+2}\right): K=a^{\perp}\right\} .
$$


The $\mathbb{C}^{\times}$-action defines fibrations and vector bundle structures on sets

$$
\left\{(l, K) \in F_{1, n}\left(\mathbb{C}^{n+2}\right): l \cap a^{\perp}=0\right\} \quad \text { and } \quad\left\{(l, K) \in F_{1, n}\left(\mathbb{C}^{n+2}\right): K \cap a=0\right\}
$$

such that the action is the scalar multiplication in the fibres and its inverse, respectively. Consider $(l, K) \in F_{1, n}\left(\mathbb{C}^{n+2}\right)$ such that $l \cap a^{\perp}=0$ and set $k:=K^{\perp}$. Let us write $l=$ $\operatorname{span}(\tilde{P}+\tilde{Q})$ and $k=\operatorname{span}(\hat{P}+\hat{Q})$ for $\tilde{P}, \hat{P} \in a, \tilde{Q}, \hat{Q} \in a^{\perp}$ and $\tilde{P}, \hat{Q} \neq 0$; without loss of generality, we can fix $\tilde{P}$ to be defined by $p=1$. Then, the action of an element $\lambda \in \mathbb{C}^{\times}$ on $(l, K)$ gives

$$
\lambda \cdot\left(\operatorname{span}(\tilde{P}+\tilde{Q}), \operatorname{span}(\hat{P}+\hat{Q})^{\perp}\right) \mapsto\left(\operatorname{span}\left(\lambda^{-1} \tilde{P}+\lambda \tilde{Q}\right), \operatorname{span}\left(\lambda \hat{P}+\lambda^{-1} \hat{Q}\right)^{\perp}\right)
$$

and the limit as $\lambda \rightarrow 0$ is $\left(a, \operatorname{span}(\hat{Q})^{\perp}\right) \in S^{1,0}$. We conclude that

$$
\left\{(l, K) \in F_{1, n}\left(\mathbb{C}^{n+2}\right): l \cap a^{\perp}=0\right\}
$$

is the trivial rank $n+1$ bundle over $\mathbb{P}\left(\mathbb{C}^{n+2} / a\right) \cong \mathbb{C P}\left(a^{\perp}\right)$. This is because for fixed $\tilde{P}, \tilde{Q}$ and a line $\operatorname{span}(\hat{Q})$, there is a unique line $\operatorname{span}(\hat{P}+\hat{Q})$, $\operatorname{such}$ that $\operatorname{span}(\tilde{P}+\tilde{Q}) \perp \operatorname{span}(\hat{P}+\hat{Q})$, and hence, projecting flags to $\tilde{Q}$ gives the trivialization of the bundle. In analogous way, we get that $\left\{(l, K) \in F_{1, n}\left(\mathbb{C}^{n+2}\right): K \cap a=0\right\}$ is the trivial rank $n+1$ bundle over $\mathbb{P}\left(a^{\perp}\right)$.

The blow-down map

$$
\phi_{0,1}:\left[\mathcal{O}(-1,0) \rightarrow \mathbb{P}\left(a^{\perp}\right) \times \mathbb{P}\left(a^{\perp}\right)\right] \rightarrow\left\{(l, K) \in F_{1, n}\left(\mathbb{C}^{n+2}\right): l \cap a^{\perp}=0\right\}
$$

is given explicitly by sending $(\tilde{Q}, \operatorname{span}(\hat{Q}))$ to the flag $\operatorname{span}(\tilde{P}+\tilde{Q}) \subset \operatorname{span}(\hat{P}+\hat{Q})^{\perp}$, and the gluing with $\mathcal{O}(0,-1)$ is given by the Hermitian product which manifests itself in the equation $\operatorname{span}(\tilde{P}+\tilde{Q}) \perp \operatorname{span}(\hat{P}+\hat{Q})$. Recall that we can use the triviality of $\mathcal{O}\left(\frac{1}{2}, \frac{1}{2}\right)$ (given by complexified Hermitian product) to identify $\mathcal{O}(-1,0)$ with $\mathcal{O}\left(-\frac{1}{2}, \frac{1}{2}\right)$ and $\mathcal{O}(0,-1)$ with $\mathcal{O}\left(\frac{1}{2},-\frac{1}{2}\right)=\left(\mathcal{O}\left(-\frac{1}{2}, \frac{1}{2}\right)\right)^{*}$. It is straightforward to check that after this identification, the gluing is $t \mapsto t^{*}$, as required in $\mathrm{qFK}$.

\section{C-map}

As already observed in [9] for $S$ Kähler-Einstein, the presented construction of quaternionKähler metric extends the Hitchin twistorial description of c-map construction ([15], see also $[1,2,14,22]$ and [11] for non-metric case) by allowing the fixed points of the $S^{1}$-action.

Indeed, let $Z$ be a quaternion-Kähler manifold obtained by qFK from a Kähler manifold $S$ and let $\mathscr{D}^{1,0}$ be the divisor from Lemma 1 and $\mathscr{D}^{0,1}$ its conjugate (corresponding to the complex structure $-I$ ). Then, the $\mathbb{C}^{\times}$quotient of the total space of the divisor bundle of $\mathscr{D}^{1,0}-\mathscr{D}^{0,1}$ over $Z$ is the hyperkähler manifold obtained by standard Feix construction from $S$. This has been shown in the proof of Theorem 6 from [9] for $S$ Kähler-Einstein and the argument extends to our situation. As a consequence, we obtain the following theorem.

Theorem 6 Let $S$ be a real-analytic Kähler manifold and $(\mathscr{L}, \nabla)$ a holomorphic line bundle with a real-analytic unitary connection whose curvature is proportional to the Kähler form. Let $M$ be a quaternion-Kähler manifold obtained by the qFK construction form $(S, \mathscr{L}, \nabla)$ and $\hat{M}$ the hyperkähler manifold obtained by the standard Feix construction from $S$. Then, away from $S$ manifolds $M$ and $\hat{M}$ are related by the Haydys hyperkähler/quaternion-Kähler correspondence. 
Acknowledgements The author would like to thank Jarosław Buczyński and David Calderbank for helpful comments and Vincente Cortés for his remark about necessity of Kähler-Einstein condition. This work is supported by the National Science Center, Poland, project "Complex contact manifolds and geometry of secants", 2017/26/E/ST1/00231, by the Grant 346300 for IMPAN from the Simons Foundation and the matching 20152019 Polish MNiSW fund.

Open Access This article is distributed under the terms of the Creative Commons Attribution 4.0 International License (http://creativecommons.org/licenses/by/4.0/), which permits unrestricted use, distribution, and reproduction in any medium, provided you give appropriate credit to the original author(s) and the source, provide a link to the Creative Commons license, and indicate if changes were made.

\section{References}

1. Alekseevsky, D.V., Cortés, V., Mohaupt, T.: Conification of Kähler and hyper-Kähler manifolds. Commun. Math. Phys. 324(2), 637-655 (2013)

2. Alekseevsky, D.V., Cortés, V., Dyckmanns, M., Mohaupt, T.: Quaternionic Kähler metrics associated with special Kähler manifolds. J. Geom. Phys. 92, 271-287 (2015)

3. Alekseevsky, D.V., Marchiafava, S.: Quatemionic-like structures on a manifold: note I. 1-integrability and integrability conditions. Rend. Mat. Ace. Lincei 4, 52-61 (1993)

4. Alekseevsky, D.V., Marchiafava, S.: Quaternionic structures on a manifold and subordinated structures. Ann. Mat. Pura Appl. IV CLXXI, 205-273 (1996)

5. Alekseevsky, D.V., Marchiafava, S.: Almost complex submanifolds of quaternionic manifolds. In: Steps in Differential Geometry, Proceedings of the Colloquium on Differential Geometry, 25-30 July. Debrecen, Hungary (2000)

6. Alekseevsky, D.V., Marchiafava, S.: Hermitian and Kähler submanifolds of a quaternionic Kähler manifold. Osaka J. Math. 38, 869-904 (2001)

7. Battaglia, F.: Circle actions and Morse theory on quaternion-Kähler manifolds. J. Lond. Math. Soc. 59, 345-358 (1999)

8. Bielawski, R.: Complexification and hypercomplexification of manifolds with a linear connection. Int. J. Math. 14, 813-824 (2003)

9. Borówka, A., Calderbank, D.: Projective geometry and the quaternionic Feix-Kaledin construction. Trans. Am. Math. Soc. 372(7), 4729-4760 (2019)

10. Calderbank, D.M.J., Eastwood, M., Matveev, V. S., Neusser, K.: C-projective geometry. Mem. Am. Math. Soc., to appear. arXiv:1512.04516

11. Cortés, V., Hasegawa, K.: The quaternionic/hypercomplex-correspondence. arxiv.org:1904.06056, eprint

12. Feix, B.: HyperKähler metrics on cotangent bundles. J. Reine Angew. Math. 532, 33-46 (2001)

13. Galicki, K.: A generalization of the momentum mapping construction for quaternionic Kähler manifolds. Commun. Math. Phys. 108, 117-138 (1987)

14. Haydys, A.: HyperKähler and quaternionic Kähler manifolds with S1-symmetries. J. Geom. Phys. 58, 293-306 (2008)

15. Hitchin, N.J.: On the hyperkähler/quaternion Kähler correspondence. Commun. Math. Phys. 324, 77-106 (2013)

16. Hitchin, N.J.: Manifolds with holonomy U*(2m). Rev. Mat. Complut. 27, 351-368 (2014)

17. Kaledin, D.: Hyperkähler metrics on total spaces of cotangent bundles. In: Kaledin, D., Verbitsky, M., (eds.) Hyperkähler Manifolds, Math. Phys. Series 12, International Press, Boston (1999)

18. Kobayashi, T.: Holomorphic vertical line bundle of the twistor space over a quaternionic manifold. J. Math. Soc. Jpn. 52, 485-499 (2000)

19. LeBrun, C.R.: Spaces of complex null geodesics in complex-Riemannian geometry. Trans. Am. Math. Soc. 278, 209-231 (1983)

20. LeBrun, C.R.: Quaternionic-Kähler manifolds and conformal geometry. Math. Ann. 284, 353-376 (1989)

21. LeBrun, C.R., Salamon, S.M.: Strong rigidity of positive quaternionic Kähler manifolds. Invent. Math. 118, 109-132 (1994)

22. Maciá, Ó., Swann, A.F.: Twist geometry of the c-map. Commun. Math. Phys. 336, 1329-1357 (2015)

23. Pedersen, H., Poon, Y.S.: Twistorial construction of quaternionic manifolds. In: Proceedings of the Sixth International Colloquium on Differential Geometry, pp. 207-218, Santiago de Compostela (1988)

24. Salamon, S.M.: Quaternionic Kähler manifolds. Invent. Math. 67, 143-171 (1982)

25. Salamon, S.M.: Differential geometry of quaternionic manifolds. Ann. Sci. Ecole Norm. Supér. 19, 31-55 (1986) 
26. Wolf, J.A.: Complex homogeneous contact manifolds and quaternionic symmetric spaces. J. Math. Mech. 14, 1033-1047 (1965)

Publisher's Note Springer Nature remains neutral with regard to jurisdictional claims in published maps and institutional affiliations. 